\title{
A review of thrust-vectoring in support of a V/STOL non-moving mechanical propulsion system
}

Review Article

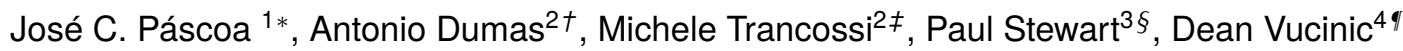 \\ 1 University of Beira Interior, \\ CAST-Center for Aerospace Science and Technology \\ Dep. Electromechanical Engineering \\ Cal. Fonte do Lameiro, 6201-001 Covilhã, Portugal \\ 2 Università degli Studi di Modena e Reggio Emilia, \\ Dipartimento di Scienze e Metodi dell'Ingegneria \\ Via Amendola 2, Pad. Morselli, 52122 Reggio-Emilia, Italy \\ 3 University of Lincoln, \\ Institute of Power and Energy, \\ Brayford Pool, Lincoln, LN6 7TS, United Kingdom \\ 4 Vrije Universiteit Brussel, \\ Department of Mechanical Engineering \\ Triomflaan 43, B-1050 Brussels, Belgium
}

Received 08 April 2013; accepted 20 June 2013

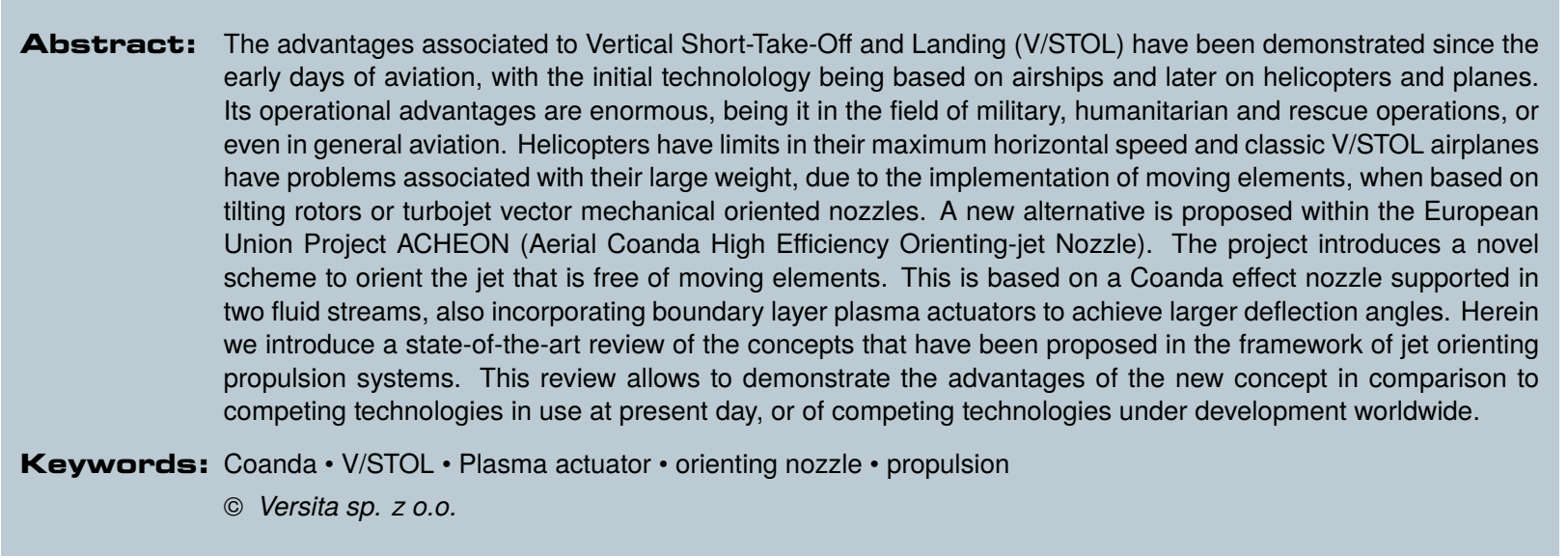




\section{Introduction}

The specific mission profiles associated to military and humanitarian operations have for many years asked for the development of V/STOL air vehicles. Also, the possibility to operate regular civil transport operations in limited air fields can benefit from this technology. Yet, and for many years, this technology was a synonymous of larger weight, reduced speed and large maintenance costs. The performance of air-vehicles can be defined and computed in a number of ways, it can be considered as the range a plane can travel, the payload it can carry, or the superior manoeuvrability of the system.

Diverse systems have been proposed to address the subject of V/STOL for air-vehicles. First, we must recognize the superior characteristics of fixed wing aircraft to achieve high speeds and, second, we can assume that the most adequate method to develop V/STOL air-vehicles is certainly based on thrust vectoring. Further, the use of thrust vectoring enables to expand the flight envelope beyond that of an aircraft using classic engine nozzles. In particular, for military mission profiles it can introduce an extension on aircraft controllability at high post-stall angles of attack, a situation where classic lifting surfaces loose their ability to provide control on the aircraft, creating what is called super manoeuvrability. This technology also improves take-off and landing performance. However, the use of moving mechanical elements with their inherent complexity is a recognized classic limitation.

In order to contribute to circumvent the highlighted limitations the European Union supports Project ACHEON (Aerial Coanda High Efficiency Orienting-jet Nozzle). The project proposes a novel propulsion concept which aims to produce a radically new aircraft propulsion system, and this can be the fundamental element of future breakthrough innovation in air transport, involving both propulsion and cost reduction, permitting even an effective redesign of air vehicles for better performance.

In particular, Project ACHEON comprises a thrust vectoring propulsive nozzle named HOMER (High-speed Orienting Momentum with Enhanced Reversibility) that is supported in a patent developed at University of Modena and Reggio-Emilia [1]. The system includes also a plasma actuator PEACE (Plasma Enhanced Actuator for Coanda Effect), this allows to extend the angle of operation of the nozzle, as proposed by University of Beira

\footnotetext{
‡E-mail: michele.trancossi@unimore.it.

EE-mail: pstewart@lincoln.ac.uk

'E-mail: Dean.Vucinic@vub.ac.be
}

Interior.

The ACHEON system, comprising the integration of the HOMER and PEACE new concepts, involves both the way in which high speed streams mix and also how they interact with Coanda surfaces, thus realizing a vectoring system which can have a wide spectrum of applications, prefiguring long term advancements in aerial and naval propulsion systems by a directionally controllable fluid jet. This novel propulsive concept is interesting for its intrinsic simplicity, which can lead to long term breakthrough innovations that could involve the aerial vehicle design as a whole and also to explore radically new concepts, such as diffused propulsion systems, novel shapes and aerodynamic concepts designed to have the maximum advantage, by vectoring thrust, and to permit the future use of novel and green propulsion concepts. It may also improve aerial vehicle design both in terms of manoeuvrability and reduction of take-off and landing spaces.

The analysis of these kind of systems can be performed using analytical, experimental and CFD tools [2? ]. A great deal of work has been performed using numerical methods for analysis and design of propulsion systems, and these can be directly applied in the present nozzle configuration [4-8]. The present system can be applied to heavier than air vehicles or even to airships $[9,10]$.

The present work will review the thrust vector concept for aeronautical propulsion, in its various implementations. Further, a review of Coanda nozzzles will be introduced in order to highlight the advantages, and limitations, of this concept in comparison with other approaches. Finally, we introduce the problem of extending the effective angle of operation of the nozzle, using plasma actuators as boundary layer control techniques, and specially the use of low weight, low power, Dielectric Barrier Discharge actuators The work starts by present the past and present framework related to V/STOL technology, specially the ones related to jet lift.

\section{An historical perspective on thrust vectoring}

Generally speaking there are two common approaches to achieve thrust vectoring (TV), namely mechanical or fluidic systems. In this respect the HOMER nozzle introduces a new generation based on Coanda effect comprising two bulk co-flow streams. Albeit presenting similarities with the fluidic approach, by not using moving elements, it is a different approach in the sense that two-bulk core streams are used, instead of a large main and a very small secondary flow control stream. We will now describe the framework in which HOMER can be used for V/STOL ap- 
plications.

An historical overview related to aircraft V/STOL technology was presented in [11]. In the first half of the previous century airships have seen a remarkable success as flying vehicles. Therefore, it is not strange to identify that among these air vehicles the thrust vectoring was first used as a control mechanism. This was the case for USS Akron and USS Macon rigid airships that used tilting propellers. This approach is still being proposed by some of present day airship projects, such as in WALRUS [12]. The use of air-jets was also proposed in those early days by Italian Engineer Enrico Forlanini in his Omnia Dir airship, built after his dead in 1931 [13].

The tilting propellers, used initially in airships, have also been proposed for airplanes, this resulted in vector thrust by rotating turboprop engine nacelles. Unfortunately, the mechanical intricacy associated with this solution have shown to be quite troublesome. A careful design must take into account the mechanics of flexible and twisting drive shafts, including gyroscopic effects. Examples of designs are the The Bell-Boeing V-22 Osprey, see Fig. 1-a), and more recently the Augusta Westland AW609, formely known as Bell/Augusta BA609 [14, 15]. Also the Eurofighter aircraft is using this technology, see Fig. 1-b). A different approach, able to vertical take-off and landing, is based on jet orienting nozzles as in the Harrier, Fig. $1-c)$.

The application of mechanical oriented nozzles, or guide vanes, to deflect the exhaust from turbofans is the most classic approach as jet vector thruster, see Fig. 1-c). It was very successful, and able to attain deflections of up to 90 degrees, at the expense of using an engine that must be designed to provide the required vertical lift, instead of the lower normal flight thrust only. One of early approaches was the Bristol Siddeley BS100 engine [16]. Another is the double flux reactor in Pegasus engine, that is used to equip the Harrier aircraft, and comprises four coupled turning nozzles that redirect the flow towards the ground for take-off and landing. In Pegasus engine the by-pass air is rejected in the two front nozzles while the hot gases are rejected by the two rear nozzles. This engine comprised several improved versions developed between 1960 and until 1970. Besides V/STOL we can also look at manoeuvrability as a target, for these the success of thrust vectoring nozzles has been many times recognized as in the Lockheed Martin/Boeing F-22 Raptor. In this case the thrust angle is between $\pm 20^{\circ}$. Still, it is generally assumed that around $30 \%$ of the weight of the F-22 Raptor engine is originated from the moving mechanical thrust vectoring mechanism [17]. These airplanes use mainly two dimensional vectoring for the pitching axis [10]. More recently, and for extending manoeuvrability opera-
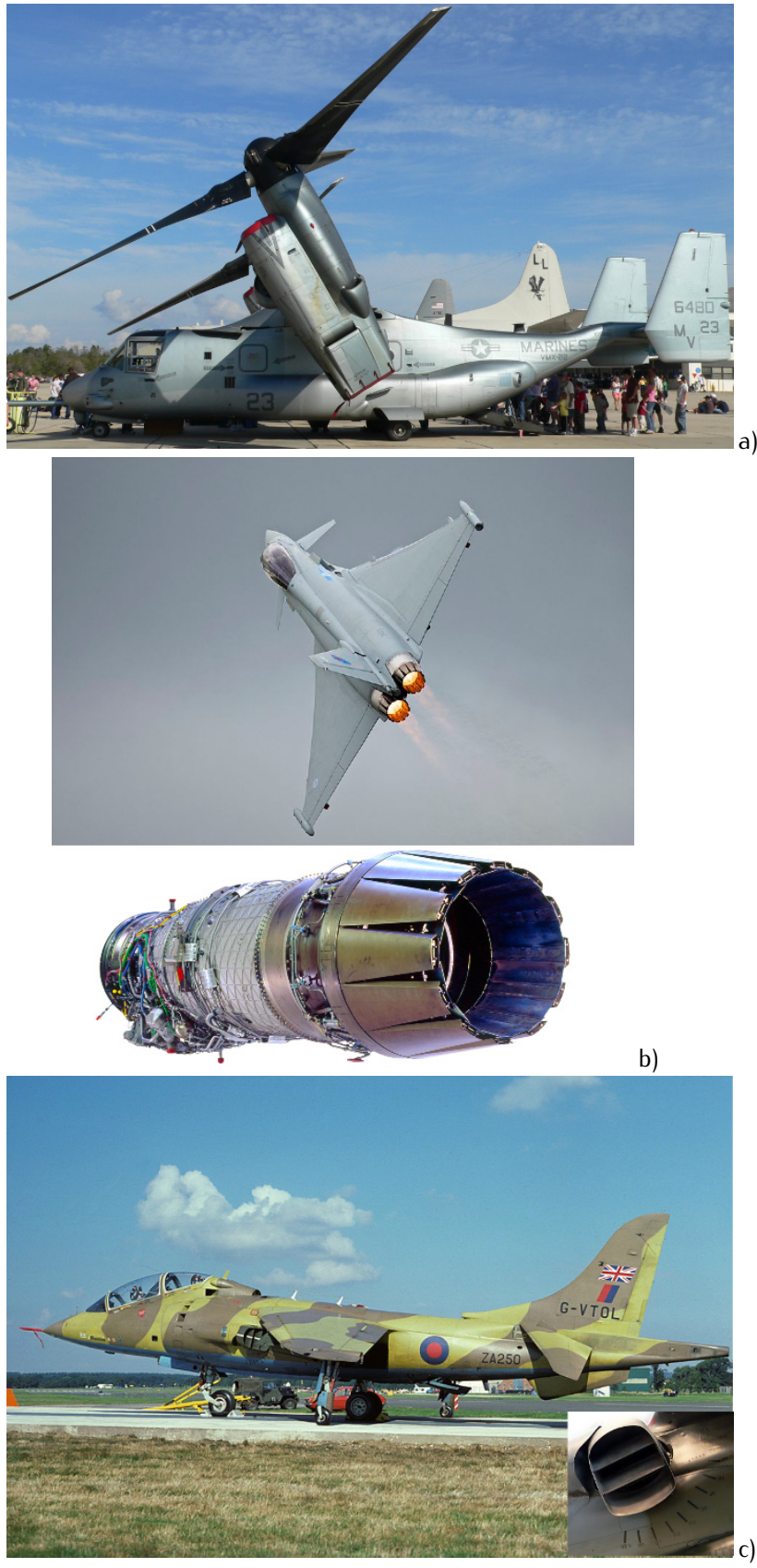

Figure 1. Examples of thrust vectoring technology in use today. a) The Bell-Boeing V-22 Osprey using a tilting rotor concept (source: obtained at NAS Pensacola). b) The modern EJ200 engine system, installed in the Eurofighter Typhoon aircraf (source: under permission of EUROJET Turbo $\mathrm{GmbH}$ ). c) Harrier fighter, including the detail of the jet orienting nozzle (source:obtained at Brooklands Museum and London RAF Museum). 
tions, also three dimensional vectoring, both for pitch and yaw axes, have been developed such as in the RockwellMesserschmitt-Bölkow-Blohm X-31 [18]. The Eurofighter Typhoon aircraf also uses this technology as seen in Fig. 1-b). More strange configurations were also proposed, as is the case for a vehicle having an umbrella type of wing with air blown from top to bottom, in order to generate lift for hovering and forward flight $[19,20]$.

\subsection{Operational considerations}

It seems clear that the V/STOL design should not compromise the cruise performance. This means that a smooth aerodynamic shape is required, in order to minimize parasitic drag, by using a reasonable span with adequate load distribution. The cruise performance must also not compromise the operation in the low speed range or at hover. It is known that a lifting system that imparts a high downwash velocity has a reduced efficiency, due to the fact that engine power varies as the cube of the air velocity. This makes clear that a large rotor propeller is more efficient to produce hover, albeit it can compromise high speed operation in cruise.

However, to correctly measure STOL performance we should rely on the usefulness of a V/STOL concept as the potential improvement in payload and range when the air vehicle is operated in STOL conditions. This productivity is heavily dependant on the configuration. The tiltwing (ex: V-22 Osprey) attains good STOL performance because it also makes use of the propeller slipstream to increase wing and flap lift. This is not the case for vehicles that put the propellers downstream of wings, since they loose the ability to effectively vector the slipstream. Another aspect is that, for jet-thrusters, a proper placement of the inlet and exhaust locations, of the lift generating system, is very important. Also, care should be exercised with the increase in momentum drag associated to the turning of the inlet flow, since it can limit forward speed in the transition, or increase take-off roll.

The transition from powered lift into conventional lift must be analysed with great care when developing any V/STOL configuration. In fact, it is necessary to provide adequate security margins in airspeed or flightpath angle when designing the drag and thrust relationships. The VFW VAK 191B airplane (produced by Vereinigte Flugtechnische Werke, in cooperation with FIAT) was based on rotating nozzle jet lift. It presented many problems since it could barely accelerate from powered lift into conventional lift, mainly due to the high induced drag and stall speed, that were compromised by the small span and wing area. In what relates to tilting-wing, when in transition from conventional flight into powered lift, the descent performance can be severely limited by wing and duct stall when the propulsive thrust is reduced, as in V-22 Osprey and similar. This concept is also very sensitive to gusts, in particular when the tilt wings are set at $90^{\circ}$. This also means that a plane that is gust sensitive cannot be expected to hover precisely. A drawback of tilt-rotor concepts.

Another operational aspect is related to the flow environment created by the propulsion system when in hovering or in low speed operation. Actually, the downwash flow from the powered lift and propulsion system can introduce hot-gas ingestion, and additional induced forces and moments on the air vehicle, or even runway (burn) deterioration. Flow ingestion is dependant on inlet flow and temperature, that can lead the compressor to work in stall or surge. There is a critical forward speed at which ingestion reaches a maximum, this one is strongly dependant on the configuration. Induced moments and pressures, more severe near ground, can produce problems related to lift and stability. Care should be exercised regarding lift-loss, that is usually more important when lift jets are close together and interact with fountain effect. Also, roll instability due to a reflection of exhaust flow from the ground into the horizontal tail, or wing surfaces, and control power reductions can compromise operation.

It is well known that V/STOL air vehicles present very complex control system requirements when compared with classic aircraft. These affect static and dynamic stability, the trim characteristics and flightpath control. A detailed description of these problems is presented in [21]. This path of research evolved over the years as can be seen in $[22,23]$.

\subsection{V/STOL implications in weight - The quest for a non-moving parts vector thruster}

For powered lift conditions, as is the case in the initial and final parts of the mission profile for V/STOL operations, the thrust to weight ratio is very important, and there is a strong need to reduce the aircraft mass in comparison to Classic Take-Off and Landing (CTOL). In Fig. 2 we describe the mass breakdown of two aircraft's, a VTOL and a CTOL, both having similar mission role and performance.

For the VTOL we must notice 3 main aspects; a) the structure mass fraction is lower than usual, and this is the result of a mandatory configuration economy, as a result of a continuous development effort, b) a larger energy source power-plant in comparison with classical, since a higher thrust to weight ratio is needed for this configuration, c) a smaller fuel fraction, this demonstrates the problems associated to the lack of volume to store fuel in the small wing, since these are not designed to take-off and landing, there is also a larger engine located centrally that 


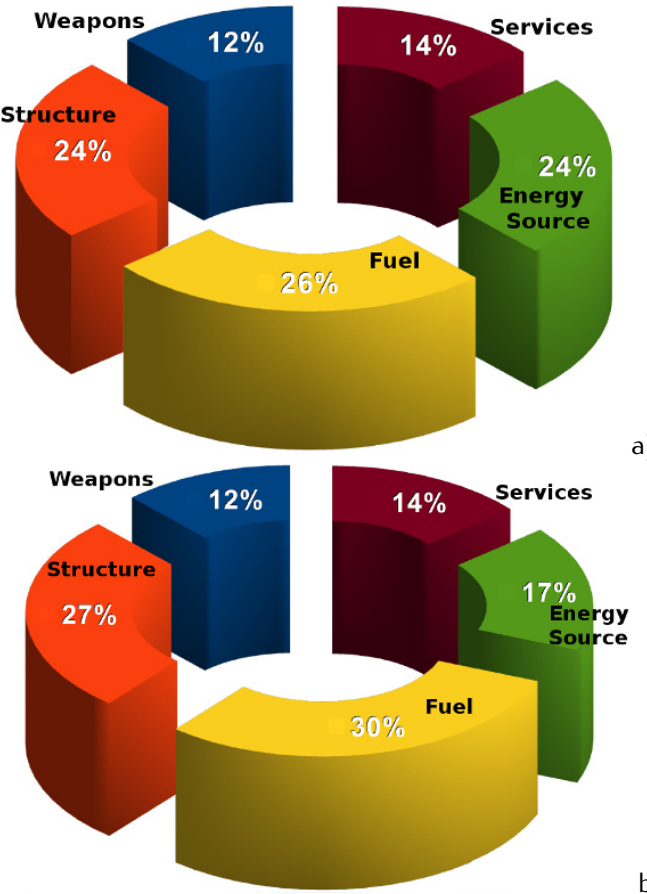

b)

Figure 2. Comparison of mass breakdown for two military planes with similar combat profile. a) Values obtained for a VTOL Harrier. b) Results obtained for a CTOL Skyhawk airplane. Marked differences between the fuel and energy source (powerplant) can be identified between the two.

limits the options for fuel tank positioning.

The implications in fuel weight have also a strong connection to the fact that the aircraft must provide a high thrust for VTOL, as a result the specific fuel consumption is not very economically throttled for the forward flight conditions. In order to partly mitigate this problem engine reheat is usually only used in forward flight, and not in powered lift conditions, but continuous reheat can also introduce another class of problems.

The number of aero-engines on-board, and their characteristics, strongly influences the final design of the airplane. Initial designers working in this field have proposed to use lightweight engines that should be only dedicated to provide lift. We can even recall the additional fan, for pure vector thrust, applied in the ancient Omnia Dir airship by Italian Engineer Enrico Forlanini, and even some recent proposals [9]. However, it is nowadays understood that there is an optimum ratio of the aero-engine, for dedicated lift, in relation to the overall propulsion weight. The minimum value depends on the requirements of the mission profile. For a close air combat mission the minimum value occurs when the powered lift engines provide $50-60 \%$ of the overall thrust installed on-board. This figure is computed by taking only into account weight, and not count- ing on financial cost limitations. However, in recent years it was considered that close air combat aircrafts should have higher agility, to insure self defence. This implies that the design should approach the one for ground air combat mission, and in this case the most optimal solution goes into the direction of $0 \%$ dedicated engine thrust. We can thus conclude that the integrated lift and cruise engine design is the one that best satisfies the requirements, by providing wide mission range performance. The ACHEON concept goes into this direction, by providing an integrated lift and cruise system.

\subsection{Thrust vector classification}

The diverse thrust vector systems can be classified according to the concept of control, see Fig. 3. The two major systems, as described previously, are the ones based on mechanical and on fluidic control concepts. The HOMER nozzle is based on non-moving mechanical parts and does not include a classic fluidics control strategy, so we have classified it in a special section.

Tilt-rotors are the most classic approach, but this kind of propulsion system is not suitable for high speed flight, because wave drag presents a fast increase at the tip of the rotor propellers. They also present extra mechanical complexity that will introduce problems of maintenance, besides the associated weight penalty. Therefore, to achieve larger horizontal velocities light weight mechanical vectorized nozzles are used, as in the case of vehicles using annular thrust vector nozzles. These are mostly used to achieve superior manoeuvrability in flight conditions and have limited deflection angles. Alternatively mechanical controlled jet impingement nozzles can extend the vector angle to $90^{\circ}$, as proposed for V/STOL aircraft.

Conversely to mechanical thrust vectors the fluidic thrust vector uses a fixed geometry. They use a secondary flow to control the main exhaust flow-stream, and in this way redirect the flow at or near the exit plane. In this regard a wide range of concepts have been proposed, such as; shock vector control, sonic throat skewing, synthetic jet actuator and co-flow or counter flow nozzles, see Fig. 4. Experimental tests concluded that the main flow can be deflected up to $15^{\circ}$. They are also beneficial because they have around 50\% lower mass and cost as compared to mechanical controlled nozzles. Further, their inertia is lower thus making them faster to actuate resulting in a stronger control response. Also, the complexity is reduced because they require a simpler mechanical system with no moving parts, having also reduced radar cross section for stealth properties [24].

The ACHEON system bears some similarity with the coflow thrust vectoring, in the sense that this later also ap- 


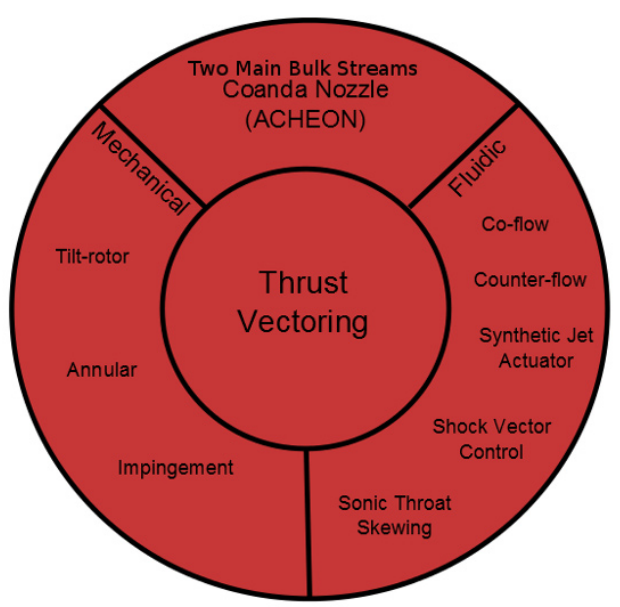

Figure 3. Classification of thrust vector systems proposed for air vehicles.
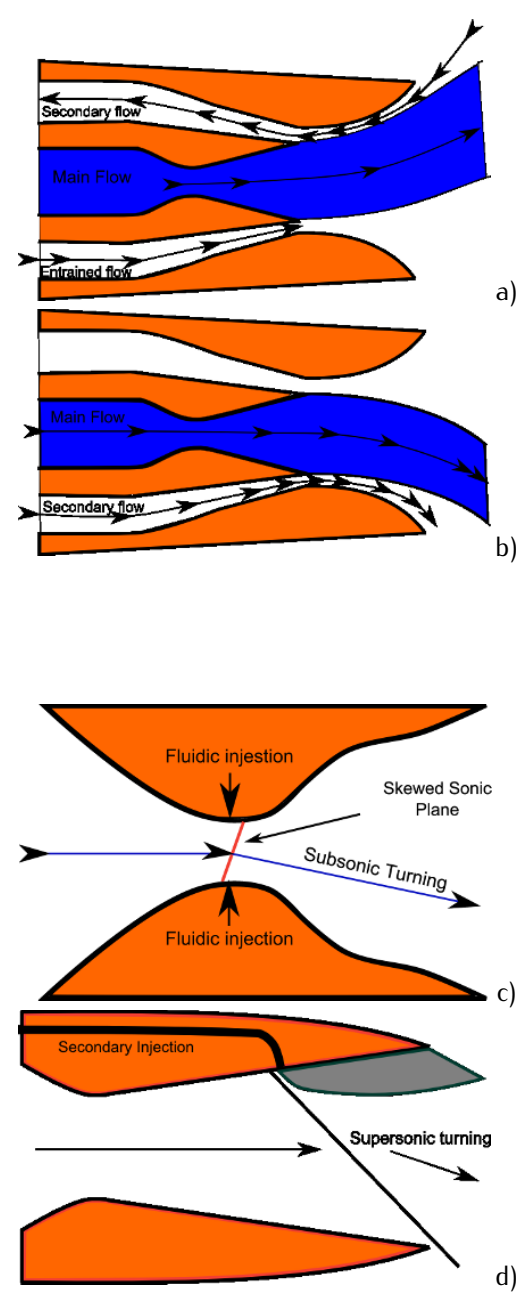

Figure 4. Fluidic vector thruster control. a) Counter flow nozzle. b) Co-flow nozzle. c) Throat shifting nozzle. d) Shock vector control. plies Coanda effect to control the deflection angle. However, the HOMER nozzle is not a fluidic based system. In Fig. 4-b) we can see that a low pressure is generated, by means of the adherence of a secondary flow to the a curved surface, entraining also a deflection of the main flow. In the counter-flow arrangement, Fig. 4-a), a vacuum is provoked on a slot that is surrounded by a suction collar around the main flow. A stream of reverse flow is created near the wall and an effect similar to the co-flow system entrains the main flow. These systems are mostly applicable to subsonic conditions, since their effect in supersonic flow can become unstable, in particular due to area rule change [25].

For high Mach numbers the concepts that became more popular are those presented in Fig. 4-c)-d). The throat shifting control system is based on a converging-diverging nozzle. Initially, the shock at the throat is a normal shock but, by applying a fluidic injection before the flow became supersonic the shape of the post throat shock is skewed, without significant loss of thrust performance. The application of the injection in the subsonic region implies that the losses are minor, thus making this system one of the most promising ones from the point of view of thrust efficiency. Another concept is based on shock vector control, by injecting a secondary control flow into the throat of a diverging section of the nozzle. In this case the formation of the flow is modified by a certain angle, since the secondary injection creates a virtual pressure ramp affecting the main flow. The main flow is deflected due to the orientation of the oblique shock. To obtain maximum deflection we must ensure that the shock does not impinge on the opposite nozzle wall, since in case the flow impacts on the wall it will be reflected thus compromising system operation [26].

Besides these canonical concepts, some authors proposed to combine the methods in order to benefit from the advantages of each [27]. The throat-shifting method is the most efficient, but requires the ability to control the throat area under different working conditions, which can be cumbersome. A large deflection angle is obtained in the shock vector control method, with its simple geometry, but can present problems associated to shock impingement and reflection The co-flow control system is the least thrust efficient method, and presents problems related to control reversal when low injection mass flows are applied. The alternative of using a counter flow system requires the availability of a suction system to produce the secondary stream

In order to circumvent the problems associated to fluidic thrust vector systems, and benefit from the absence of moving parts, the ACHEON system was introduced [28-30]. The HOMER nozzle bears some resemblance with the co- 


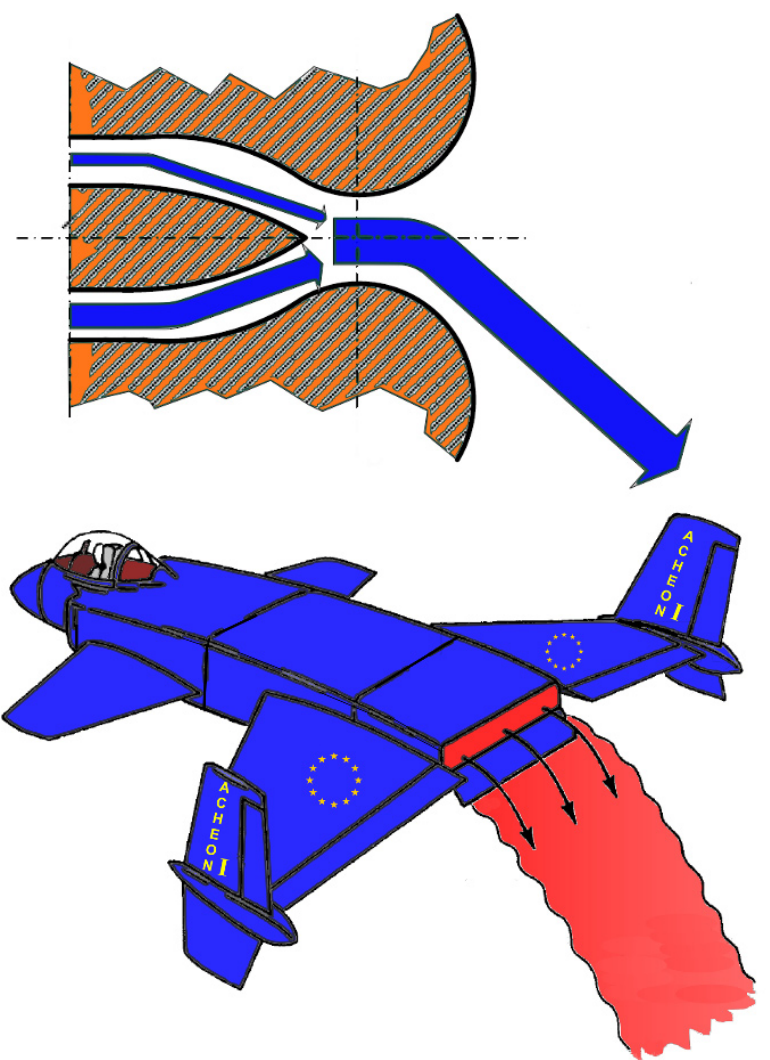

Figure 5. The HOMER nozzle comprising two main streams that, when in differential mass flow operation, will cause the thrust vectoring of the nozzle. The system can be incorporated on an airplane to achieve STOL, or even eventually VTOL for alternative configurations.

flow system, but it is not a fluidic, and in this sense it will be less prone to problems associated with the injection of reduced mass flows. In this case there is no secondary fluidic stream, but only two co-flow streams whose differential mass flow allows to control the thrust vector angle, see Fig. 5.

The performance of the HOMER thrust vectoring is strongly dependent upon the two core flow stream properties and also on the specific nozzle geometry. In this aspect the curved wall shape, besides the exit slot aspect ratio, are the main parameters that affect the vector angle [31].

\section{Coanda effect research applica- ble to vector nozzle thrusters}

If we intent to study the behaviour of jets and wakes, and their interaction with a V/STOL airframe, we need to start by an understanding of the fundamental research that has been developed for simpler jets. Yet, if we extrapolate directly that analysis to try to understand the effects on a real aircraft, we may be committing mistakes, since the properties of jets produced by aero-engines are very different from the ones in laboratory jets. The vast majority of data available was obtained using small scale experiments. In many cases they are lower than a Reynolds of $1 \times 10^{6}$, but in practical applications the Reynolds numbers computed for the jets are much larger. Here, the influence of Mach number must also be considered as a representative parameter. Another effect, maybe less important if we use electric drive turbofans, is the change in thermodynamic properties when we compare the model tests to the jets discharged by V/STOL aircraft. Extreme care should be taken when reasoning on the basis of cold jets having low initial turbulence, and starting from an almost uniform velocity profile, and in the absence of jet swirl. Since in practice the jets emanating from aero-engines are at elevated temperature, have high turbulence levels, and a non-uniform velocity profile sometimes with velocity components normal to the flow direction [32].

The concept of Coanda effect - the phenomenon of jets adhering to and flowing around nearby solid boundaries - and the corresponding advantages of its use have been studied for many years. Besides its use in propulsion systems, the concept was also proposed for industrial applications, such as in the control of spray manipulation for commercial plasma spray gun, or in combustion systems [33-35].

Most of the research work was carried out using incompressible air jets, exiting from rectangular orifices issuing tangentially to and flowing along the surface of a curved wall into quiescent ambient air. In [36], for example, an experimental study of jet separation is presented. It is demonstrated that the three-dimensional curved wall jet is different in its average properties from its two dimensional counterpart. Two main tendencies can be identified in this kind of experiment; a free jet flow and the specific Coanda flow. This kind of behaviour occurs when we use exit orifices with small aspect-ratio. Further, in three dimensional curved wall jets the maximum velocity decay can be characterized by three regions. This maximum velocity decay rate is dependent on the exit orifice aspect ratio, exception made for a potential core region. Also, the curved wall jet presents a different growth behaviour in comparison with other three-dimensional jet flows.

\subsection{The concept of Coanda nozzle}

There are two main problems that must be considered in the Coanda jet deflection around small radii curves, for $R / t \leq 10$, based on the jet orifice width. The first one is 
related on how to initially attach the jet, the second is on how to keep the deflection by delaying separation.

Regarding the first problem, we must recognize that the inertia of the exit jet will try to resist deflection. The inertial force, centripetal acceleration times mass, $\frac{\rho V^{2}}{R}$ increases as the radius of the turn decreases, preventing attachment when larger values of inertia force are obtained, i.e. when they are larger than the initial radial pressure gradient $\partial P / \partial r$. Since it is this later that can deflect the jet towards the surface. In the literature, the attachment limit conditions have not yet been established with full confidence. Physically, as the jet exits from the slot the curved wall surface pressure is below atmospheric pressure, causing the jet to move towards the curved wall surface.

If a jet is attached to a flat surface, and in the absence of external disturbances, it will remain attached. But this is not true for a curved surface, since it will sooner or later separate, due to increase of pressure at the wall that equates the atmospheric pressure. The viscosity entrains the development of a boundary layer near the curved surface, together with a mixing layer in the outer radial position that connects to the surrounding fluid (being it quiescent or a main jet). This later is dragged and causes a separation of the boundary layer. Also, since the jet thickness increases, it occurs a reduction in secondary jet average velocity, that also contributes to separation, since the pressure will increase at the wall.

Let us consider the two-dimensional radial momentum equation in polar coordinates, under the assumption that the viscous terms are small compared to inertial effects in a free jet. The geometry is based on a similarity with Fig. 6-a). As the jet flows around the curved wall, the inertia force is balanced by the radial pressure gradient, mathematically we can write,

$$
\frac{\partial P}{\partial r}=\frac{\rho V^{2}}{R}
$$

We can identify this equation in a more physically sound manner if we consider it as $\frac{\Delta P}{\delta r}=\frac{\rho V^{2}}{R}$, here $\delta$ is the jet thickness. In a first order approach, the pressure at the curved Coanda surface can be computed from,

$$
P(\theta)=P_{\infty}-\frac{T \theta}{R(\theta)}
$$

Here $T$ is the local momentum of the air jet. The wall friction will reduce the jet thrust when it flows around the curved wall surface, also the average radius of curvature is increased by the effect of mixing of the outer jet boundary with the surrounding air. The combined effect of these two physical actions induce an increase in surface pressure. This will cause the separation of the jet, sooner or later depending on the severity of the formed pressure gradient. Albeit no final clarification is presented in the literature regarding the prediction of the separation point, it is generally assumed that it depends on the initial jet thrust, on the radius of the Coanda wall, and on the properties of the fluid. We can thus write $\theta_{\text {sep }}=f(R / t, R e, M)$. Therefore, we can conclude that the action of attachment can be explained by a pure inviscid mechanism, by considering the balance of centrifugal forces, but the viscous effects are the main cause of jet separation from the curved wall. Even if there is a theoretical value for the maximum angle that can be computed based on pure inviscid theory, it will never be reached in reality!

Experimental results, quoted in [37], demonstrate that for incompressible flow $\theta_{\text {sep }}$ increases with $R / t$ and $R e$ until a maximum value of $245^{\circ}$. For $R / t=5$ the maximum turning angle is around $170^{\circ}$.

\subsection{Weakening of Coanda effect due to 3D ef- fects}

In the classical wall jet flowfield we can describe two patterns for the flow as we travel in a direction normal to the wall. The internal part presents the characteristics of a boundary layer evolving along a surface, but further upper an external part exhibits the aspect of a free jet type of flow field. If the jet is evolving along a flat wall a smooth transition between the two parts is identified since there is no separation point.

For the case of a two dimensional curved wall jet there is a deflection of the flow by Coanda effect. The establishment of the Coanda flow is strongly dependent on maintaining mostly two dimensional flow conditions. Specially, one should prevent spanwise flow and entrainment parallel to the major axis of the orifice. This can be accomplished by using end-plates [36].

For a generic three-dimensional curved wall jet, Fig. 6a), the occurrence of spanwise and entrainment from the both ends is not a minor issue, specially as the exit jet becomes less slender, i.e. if aspect ratio assumes values around 1 . This causes a weakening of the Coanda effect, that is almost acting only on the central part of the exit jet. The outer and free jet portions, that have higher momentum than the internal part, have a tendency to maintain the primitive exit direction, barely being affected by the Coanda effect.

The wall jets have an approximately triangular separation profile on the wall. The origin is at $\theta=0$ and the apex is downstream in the centerline of the wall jet. We may thus conclude that the separation of the jet proceeds from lateral sides, spanwise, into the centerline as $\theta$ increases. The centerline separation angle $\theta_{\text {sep }}$ will depend on the 


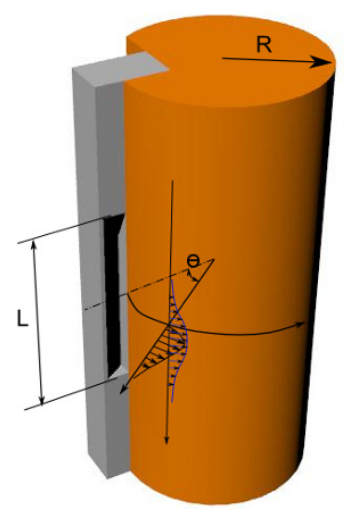

a)

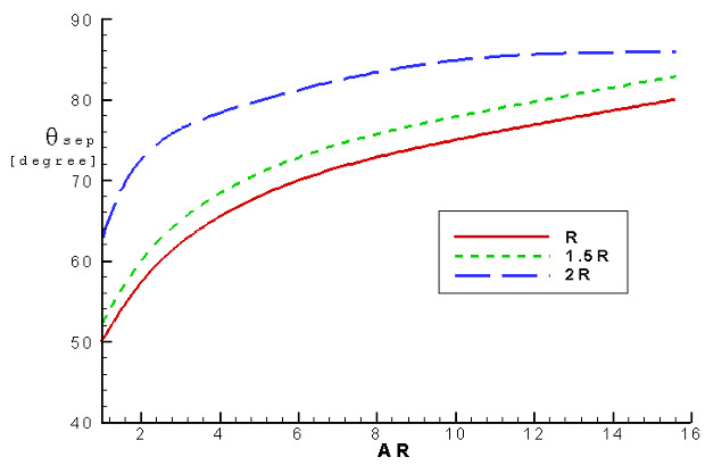

Figure 6. Schematic drawing representation of three-dimensional curved wall jet flow field a). Variation of center line separation angle with aspect ratio and radius of curvature $b$ ). Data compiled from [36].

aspect ratio $A R=L / t$, with $L$ the jet orifice length and $t$ the jet orifice width. Also the radii of curvature affects the separation angle. Figure 6-b) presents results for three values of $R$. Here we can see that the effect of radii of curvature is less important than the effect of aspect ratio, this is due to the fact that the experimental jet Reynolds number was relatively high,

$$
R e=\left[\frac{\left(P_{t o}-P_{a}\right) R(L t)^{0.5}}{\rho v^{2}}\right]^{0.5}=9.5 \times 10^{4}
$$

One can see that even with the greater $A R=15.6$, the separation obtained is of the order of $85^{\circ}$, this value is significantly lower than the values obtained in pure twodimensional curved wall jets. Globally the separation angle increases with the aspect ratio and radius of curvature. Similar results have also been demonstrated for a oscillating jet under Coanda effect [38].

We may then conclude that in 2D Coanda nozzles the three-dimensional flow effects, in the lateral sides, is deleterious for achieving a large separation angle. However,

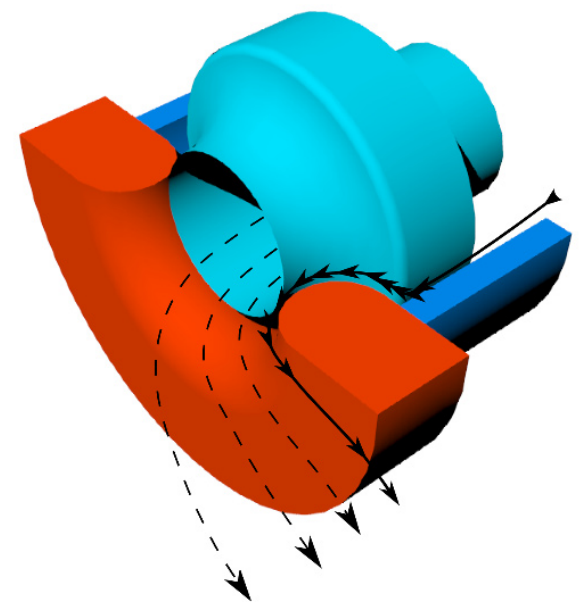

Figure 7. Scale drawing of a 3D Coanda-assisted vectoring nozzle. The application of secondary flow at one circumferential location will cause the primary jet to vector toward the control flow.

several authors have also provided designs able to create $3 \mathrm{D}$ vectoring $[25,39]$. In this sense the system will enable, b) not only to control the airplane pitching, but also to provide roll and yaw support. Figure 7 provides an example of such a system. A lateral secondary jet is activated in order to deflect the main flow into the desired direction.

The lateral secondary jet is emanating from a slot that is positioned in a defined circumferential position. This slot is able to rotate circumferentially around the main nozzle axis, in order to change the deflection angle position circumferentially. The reduced pressure effect counterbalances the dissipation of boundary-layer energy until the flow ultimately detaches from the surface. A careful design of the system is needed in order to avoid bistable or even unstable operation, that will cause an undesirable flapping of the main flow.

Several researchers have performed extensive studies on the evolution of the turbulent flow around cylinders in the conditions of Fig. 6. They demonstrate that large streamwise vortices occur in a turbulent wall jet flowing over a convex surface. The existence of these vortices is due to a centrifugal instability [? ]. They develop across the span, and they become larger with increasing distance from the nozzle. Although the longitudinal structures are not contributing to the mean spanwise distortion of the flow, they are strong enough to augment the Reynolds stresses that increase the rate of spread of the jet and its turbulent intensities [41-43]. 


\subsection{Curved jet flows associated to ejector thrust augmenters}

A jet is a stream of air which has a velocity markedly different from the main body of air being considered in a problem. Jets did not play a significant role in aerodynamics until the advent of the gas turbine for propulsion; even then, it was the propulsive force obtained, rather than the finer details of the jet flow structure, that received most of the attention. The propulsive force depended on the flow condition at the engine exit nozzle, not on what happened downstream. The advent of the jet flap, annular jet, and other similar concepts markedly changed this picture. For the first time, a jet was being used to influence the main flow field, and the characteristics of the jet, after it left the nozzle, were found to be the controlling parameters [44? , 45].

Generally speaking, the science behind the curved jet flow presents the same problems as the pure rectilinear jets, having an additional complexity that is related to the centripetal acceleration.

We define ejector as a device that uses entrainment by a main jet to apply momentum to an associated near flow. This concept was proved as efficient to increase the static thrust of turbojet engines, and it can be obtained by entraining flow from the surroundings, in the same way as a turbofan, but without the fan. This occurs when the exit jet is at a pressure lower than atmospheric. In this way thrust is augmented by accelerating a large mass of air entrained from the atmosphere. This kind of ejector can be used to increase and to deflect engine thrust. When this ejector is integrated into the wing, to create lift-propulsion systems, the exhaust air will act as a jet flap thus allowing an increase in circulation lift of the wing, improving V/STOL characteristics, see Fig. 8-a).

If we recall that HOMER Coanda nozzle also entrains nearby flow, then it should also create a thrust augmentation effect. But this will be difficult to achieve since skin friction loss in the curved wall will counter act this augmentation effect. Besides exhausting at a pressure lower than atmospheric, a second factor that determines augmentation is the performance with which the flow is diffused back to ambient from the mixing pressure. For relatively thick jets, the diffusion loss at the end of a Coanda curve can be quite high, in this way high augmentation ratios can only be obtained by keeping the jet thin. But if one keeps the jet thin the wall friction losses will also increase, and this is a drawback.

A second reason to use thin jets, if we wish to take benefit from jet thrust augmentation, is related to the quantity of environment fluid that is entrained by the jet. To achieve high entrainment ratios one requires a jet thickness which is only a few percent of the radii of curvature, but again

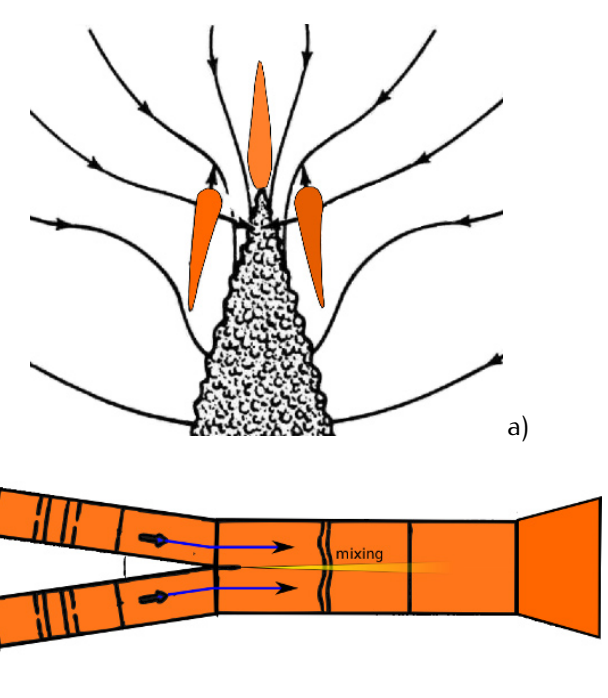

b)

Figure 8. Details affecting the thrust performance of the propulsion nozzle. a) Jet mixing in the region of reduced pressure, at primary nozzle exit, can be used to entrain fluid and increase the initial jet thrust, by increasing the accelerated amount of mass. b) A non-adequate treatment of the mixing layer, formed when two different mass streams join inside the HOMER nozzle, can enlarge the losses and reduce the performance, see also Fig. 5.

boundary layer effect become important in this case. This consideration also introduces a new aspect, being that the separation angle will also be strongly dependent up the momentum conditions of the atmospheric air. The values obtained by the vast majority of authors are for still air, but HOMER will be used in the dynamic conditions of a plane in forward flight $[47,48]$.

Another detail that must be considered, and that can affect the overall performance of these systems, is the analysis of the fluid mixing in the two internal streams, see Fig. 8-b). The mixing layers from non-parallel merging of streams was found to grow faster in the near-field and achieve self-similarity earlier than on the parallel merging case. The growth of the mixing layers was found to decrease with increasing velocity ratio. It is also found that the mixing layers spread more into the high speed region with increasing velocity ratio. Also, the splitter wake was found to have an enduring effect on the development of the mixing layer, and should be adequately designed [49].

\subsection{Problems associated with hysteresis ef- fect in supersonic Coanda Nozzles}

It is well known that the V/STOL Harrier fighter, see Fig. $1-\mathrm{c})$, has a subsonic top speed of around $1180 \mathrm{~km} / \mathrm{h}$. If we consider pure mechanical moving nozzles there is no physical limitation on the maximum speed that can be 


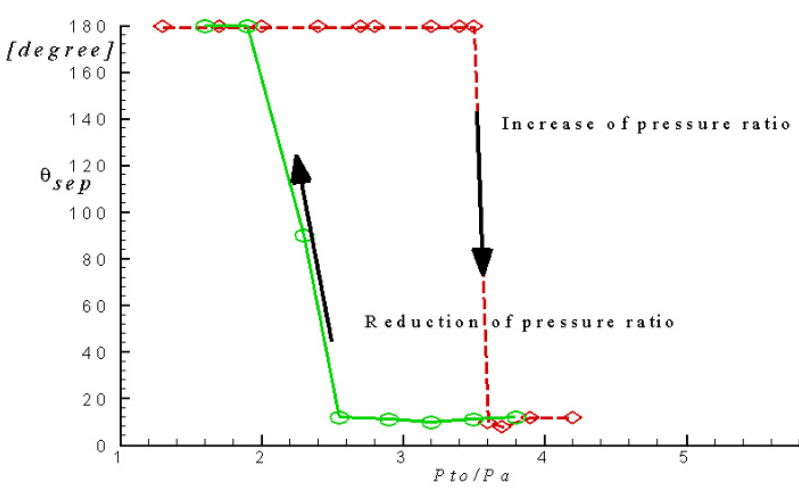

Figure 9. Hysteretic characteristics of a Coanda supersonic jet for a ratio of $R / t=5$. Data compiled from [50].

achieved by a plane. However, if we resort to a Coanda effect nozzle this is not the case.

In Fig. 9 we can see that, for a supersonic flow, there is a detachment and reattachment of the jet flow that shows some hysteresis. As described in [50] there is a reduction in the hysteresis loop when one decreases the ratio of radius to jet orifice width $R / t$. It is considered that a balance between momentum of jet, for detachment, and the entrainment rate of ambient gas into the curved jet, for reattachment, affects the hysteresis.

The reasons for the development of the hysteresis loop are related to the non-linear behaviour of the shock wave pattern created at the nozzle exit. Also, there is a formation of a separation bubble, creating a region were the turbulent kinetic energy becomes large, resulting in an effecting mixing of the jet with ambient air. This can be useful for some kind of applications, like in combustion, but the hysteresis effect renders the Coanda nozzle thruster non-controllable in the supersonic regime.

We must say that it was demonstrated, in [37], that it is possible to define the shape of a nozzle, using the method of characteristics, able to keep the flow attached for large angles. However, it will not be capable of keeping that performance for different pressure ratios, due to the occurrence of hysteresis loops.

More recently, specially designed confined counter-flow fluidic systems have been proposed that are able to present multi-axis thrust vector response for supersonic jets with no moving parts [51]. The authors claim that a continuous and proportional control of the jet deflection can be achieved up to Mach 2. This was tested to control pitching using rectangular nozzles and also for 3D control using axis-symmetric nozzles. The maximum deflection is of around $15^{\circ}$ for a careful designed pressure distribution in a $3 \mathrm{D}$ collar. The authors also recognize the destabiliz- ing effect of hysteresis, claiming that counter-flow nozzles can behave better in this respect.

\subsection{Preventing separation in Coanda Nozzles by plasma actuators}

The application of active flow control is very important in the field of aeronautical applications. Efficient flow control systems are capable of manipulating the flow to achieve certain desired effects, such as drag reduction, mitigation of noise pollution, and help increase a reducfion in stall margins on airfoils. In order to achieve these results two main techniques are used; separation control and laminar-to-turbulent transition suppression. The most popular flow control methods usually involve the use of mechanical flaps, suction and blowing techniques, piezoelectric actuators, synthetic jets, as well as MEMS devices. A detailed review on active flow control techniques can be found in [52]. Recently, the introduction of plasma actuators in the field of aerodynamics has demonstrated to be very promising to achieve flow control at reduced cost and weight. Plasma-based devices exploit the momentum coupling between the surrounding gas and plasma to manipulate the flow. Unlike other flow control techniques, such as suction and mechanical actuators, plasma actuators require low power consumption, involve no moving mechanical parts, and have a very fast frequency response that allows real-time control. For these reasons, the plasma actuator has become a very promising and attractive device in the flow control community.

Specifically, there are two methods that have been proposed to prevent the separation of Coanda jets. One concept is based on the use of multiple flat plate turning surfaces. In this concept the angular corners will introduce a tripping on the boundary layer, this will delay separation by increasing the boundary layer energy. This methodology proved not to be very efficient [53].

A second alternative that has been proposed is to create blowing exit slots on the curved surface. This technique was proved as very effective for subsonic Coanda jets.

In the context of the ACHEON Project, the HOMER nozzle will be provided with the PEACE actuator. PEACE is the acronym of Plasma Enhanced Actuator for Coanda Effect. PEACE aims to produce an active control of the Coanda adhesion to a surface by means of the DBD technology (Dielectric Barrier Discharge) which can enhance and control adhesion of the Coanda jet.

Plasma actuators can be sub-categorized into two major families: the corona discharge, and the dielectric-barrierdischarge. Recently, the most commonly used plasma actuator has become the single-dielectric-barrier-discharge (SDBD). Two electrodes are typically separated by a di- 
electric barrier made of glass, Kapton or Teflon as depicted in Fig. 10. When a high AC voltage signal, of sufficient amplitude (5-40 kV) and frequency (1-20 kHz), is applied between the electrodes the intense electric field partially ionizes the surrounding air, producing a non thermal plasma on the dielectric surface. The collisions between the neutral particles and accelerated ions generate a net body force on the surrounding fluid leading to the formation of the so called ionic wind. The body force can be used to impart the desired flow control outcome on a given fluid system. For the SDBD configuration the momentum coupling of the plasma and fluid induces an initial vortex that propagates downstream. Very promising results for the application of plasma actuators have been observed in a wide range of applications. Hanson et al. [54] were able to effectively control the transient growth instability in a Blasius boundary layer, by delaying transition through the injection of a counter-disturbance into the boundary layer using the plasma actuator, their results were obtained experimentally. Separation control has also been successfully achieved for a wide range of bodies, including a NACA 0015 airfoil, a cylinder and a hump in the presence of a turbulent boundary layer [5558]. Other applications include the control of a rotor blade wake [59], increasing the lift on a UAV [60], and noise reduction [61].

A plasma actuator consists of two offset thin electrodes that are separated by a layer of dielectric insulator material. One electrode is exposed to the air. The other is fully covered by a dielectric material. The electrode exposed to air is assumed to be loaded by a high voltage, whereas an electrode buried under the dielectric is expected to be grounded. A high voltage AC (high-amplitude (several $\mathrm{kV})$ ) and high-frequency (typically several $\mathrm{kHz}$ ) is supplied to the electrodes. This effect permit a partial ionization in the region of the largest electric potential, which usually begins at the edge of the electrode that is exposed to the air, and spreads out over the area projected by the covered electrode. The ionized air (plasma) in presence of the electric field produces an attraction/repulsion on the surrounding air. Ionized particles are accelerated and transmit their momentum, through collision, to the neutral air particles in the plasma region over the covered electrode [62]. The result is an acceleration of the air in proximity of the surface of the dielectric, see Fig. 10.

Actually, plasma actuators can be applied in a similar way as any other boundary layer control devices. However, DBD plasma actuators have a large number of advantages over other active flow control devices:

-very simple, fully electronic, no moving parts;

-operated in either steady (continuous) and unsteady (pulsed or duty cycle) modes;
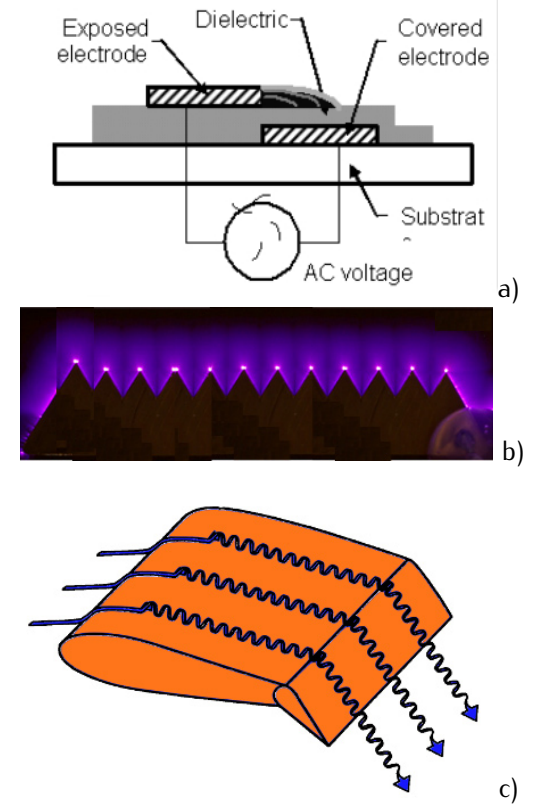

Figure 10. Boundary layer control using plasma actuator. a) Dielectric Barrier Discharge (DBD). b) Riblet of DBD actuators in sawtooth shape. $\mathrm{c}$ ) Effect of the vortex generators to control boundary layer flow.

-low power consumption (0,0067-0,0134 Watts per mm for unsteady operation);

-simple integration, maintenance and operating costs;

-they do not affect surfaces and their aerodynamic performances,

-conformability to any surface curvature;

-high mechanical resistance;

-affordability and durability;

-fast response for feedback control due to high bandwidth and possibility of closed-loop feedback control;

-possible modulation in terms of frequency and of power variations.

\section{Conclusions}

The development of a technology able to support the next generation of V/STOL aircrafts is of extreme importance to insure specific mission profiles. This kind of vehicles can also contribute to introduce new green energy technologies in the aeronautics field. This is one of the main goals of the European Union funded project ACHEON. It will also insure that European Aeronautics industry will keep its worldwide leadership in this field.

One of the greatest arguments to the development of nonmoving thrust vector aircraft systems is related to weight 
reduction. It was demonstrated that classic, mechanical moving, thrust vector systems imply a reduction in the operative range, due to the associated reduction in fuel carrying capability, along side with the increased mechanical complexity that introduces maintenance problems.

In this context, the proposal of the HOMER nozzle, that results from a patent developed by some of the team members, will certainly contribute to introduce a new insight in this research field. The system is based on two bulk differential fluid streams coupled into a Coanda effect nozzle.

The development of a complete operative HOMER nozzle was demonstrated to be dependant on the solution of specific performance issues. Among these there are problems associated to the internal losses occurring inside the system. The mixing of two streams with differential velocities implies a mixing effect that conducts to losses. This issue must be dealt with care, and specifically supported in the more recent studies that have been done on the subject. Instability effects related to Mach number and to 3D effects have been shown to strongly affect the HOMER nozzle concept inside ACHEON. It was demonstrated that a supersonic flow can introduce an hysteretic effect that compromises the stability of the flying vehicle. Also, in order to achieve a large deflection angle for the jet, the side effects of the jet development must be controlled. Since they can conduct to early separation of the flow, and in this way reduce the operative conditions of the aircraft. This aspect must be also correlated with an exit thrust augmentation geometry, in order to increase the efficiency of the propulsion system.

The present work presents a detailed review that embraces the scientific and technological aspects of the ACHEON system. Not only looking at the basic physics of Coanda effect thrusters, but by also interconnecting with the associated problems of its implementation into real air vehicles. Albeit this, the design must also take into account the structural problems associated with these concepts [64?].

\section{Acknowledgements}

The present work was performed as part of European Project ACHEON (Aerial Coanda High Efficiency Orienting-jet Nozzle) under Contract NÂž 309041 of the 7th Framework Programme.

\section{References}

[1] Trancossi M., Dumas A., Giuliani I., Bagi I., Ugello capace di deviare in modo dinamico e controllabile un getto sintetico senza parti meccaniche in movimento e suo sistema di controllo, Patent No. RE2011A000049, Italy, 2011.

[2] Vucinic D., Hazarika B., Dinescu C., Visualization and PIV measurements of in-cylinder axisymmetric ows, in SAE Technical Papers, Automotive and Transportation Technology Congress and Exhibition, 2001.

[3] Vucinic D., Hazarika B., Integrated approach to computational and experimental ow visualization of a double annular conned jet, Journal of Visualization, vol. 4, no. 3, pp. 245-256, 2001.

[4] Pascoa J., Xisto C., Goettlich E., Performance assessment limits in transonic 3D turbine stage blade rows using a mixing-plane approach, Journal of Mechanical Science and Technology, vol. 24, no. 10, pp. 20352042, 2010.

[5] Pascoa J., Mendes A., Gato L., A fast iterative inverse method for turbomachinery blade design,Mechanics Research Communications, vol. 36, no. 5, pp. 630-637, 2009.

[6] Pascoa J., Mendes A., Gato L., Redesigning annular turbine blade rows using a viscous-inviscid inverse design method, in Proc. 53rd ASME Turbo Expo 2008, vol. 6 of A, pp. 2209-2217, 2008.

[7] Pascoa J., Mendes A., Gato L., Turbine blade duty re-design by controlling lean and sweep using an innovative iterative inverse design method, in Proc. 51st ASME Turbo Expo, vol. 6, pp. 1249-1256, 2006.

[8] Pascoa J, Mendes A., Gato L., Aerodynamic design of turbomachinery cascades using an enhanced time-marching nite volume method," CMES-Computer Modeling in Engineering and Sciences, vol. 6, pp. 537-546, 2004.

[9] Smith T., Bingham C., Stewart P., Allarton R. et al., Energy harvesting and power network architectures for the multibody advanced airship for transport high altitude cruiser-feeder airship concept, Journal of Aerospace Engineering, Part G of Proceedings of the Institution of Mechanical Engineers, vol. 227, pp. 586-598, 2013.

[10] Stewart P., Gladwin D., Parr M., Stewart J., Multiobjective evolutionary-fuzzy augmented flight control for an F16 aircraft," Journal of Aerospace Engineering, Part G of Proceedings of the Institution of Mechanical Engineers, vol. 224, pp. 293-309, 2010.

[11] Anderson S. B., Historical overview of V/STOL aircraft technology, Tech. Rep. TM-81280, NASA, 1981.

[12] Ilieva G., Pascoa J., Dumas A., Trancossi M., A criti- 
cal review of propulsion concepts for modern airships, Central European Journal of Engineering, vol. 2, no. 2, pp. 189-200, 2012.

[13] Flight, pp. 289, April 1932

[14] Thomason T., Bell-Boeing JVX tilt rotor program flight test program, in AIAA Paper N 83-2726, American Institute of Aeronautics and Astronautics, 1983.

[15] Aviation international news, pp. 42, February 2012.

[16] Flight international, pp. 1014, June 1962.

[17] Aviation Week - Last Raptor Rolls Off Lockheed Martin Line, December 2011.

[18] Dorr R., Rockwell/MBB X-31, World Air Power Journal, vol. 24, pp. 34-47, 1996.

[19] Saeed B., Exploring the Aerodynamic Characteristics of a Blown Annular-Wing for V/STOL Aircraft. PhD thesis, Brunel University, 2010.

[20] Saeed B., Gratton G., Mares C., A feasibility assessment of annular winged VTOL flight vehicles, The Aeronautical Journal, vol. 115, pp. 683-692, 2011.

[21] AGARD, V/STOL handling i-criteria and discussion, Tech. Rep. AGARD-R-577-70, Advisory Group for Aerospace Research \& Development, 1970.

[22] Moralez E., Merrick V., Schroeder J., Simulation evaluation of an advanced control concept for a V/STOL aircraft, Journal of Guidance Control and Dynamics, vol. 12, pp. 334-341, 1989.

[23] Naldi R., Marconi L., Optimal transition maneuvers for a class of V/STOL aircraft, Automatica, vol. 47, pp. 870-879, 2011.

[24] Strykowski P., Krothapalli A., An experimental investigation of active control of thrust vectoring nozzle flow fields, tech. rep., The University of Minnesota, 1993.

[25] Mason M.,. Crowther W., Fluidic thrust vectoring of low observable aircraft, in CEAS Aerospace Aerodynamic Research Conference, pp. 1-7, 2002.

[26] Wing D., Static investigation of two fluidic thrustvectoring concepts on a two-dimensional convergentdivergent nozzle, Tech. Rep. TM-4574, NASA Langley Research Center, 1994.

[27] Porzio A., Characteristics of a Confined Jet Thrust Vector Control Nozzle. PhD thesis, Air Force Institute of Technology (USA), 1984.

[28] Trancossi M., Dumas A., A.C.H.E.O.N.: Aerial Coanda High Efficiency Orienting-jet Nozzle, in SAE Aerotech 2011, no. SAE Technical Paper 2011-012737, 2011.

[29] Trancossi M., An overview of scientific and technical literature on coanda effect applied to nozzles, in SAE Aerotech 2011, no. SAE Technical Paper 2011-012591, 2011.

[30] Trancossi M., Dumas A., Coanda synthetic jet deflec- tion apparatus and control, in SAE Aerotech 2011, no. SAE Technical Paper 2011-01-2590, 2011.

[31] Sagha F., Banazadeh A., Coanda surface geometry optimization for multi-directional co-flow fluidic thrust vectoring, in Proceedings of ASME Turbo Expo 2009: Power for Land, Sea and Air, no. GT200959715, 2009.

[32] AGARD, Special course on V/STOL aerodynamics, Tech. Rep. AGARD-R-710, Advisory Group for Aerospace Research \& Development, 1984.

[33] Mabey K., Smith B., Whichard G., McKechnie T., Coanda-assisted spray manipulation collar for a commercial plasma spray gun," Journal of Thermal Spray Technology, vol. 20, no. 4, pp. 782-790, 2011.

[34] Kim H., Rajesh G., Setoguchi T., Matsuo S., Optimization study of a coanda ejector, Journal of Thermal Science, vol. 15, no. 4, pp. 331-336, 2006.

[35] Vanierschot M., Persoons T., Van den Bulck E., A new method for annular jet control based on cross-flow injection, Physics of Fluids, vol. 21, pp. 025103-1025103-9, 2009.

[36] Patankar U., Sridhar K., Three-dimensional curved wall jets, Journal of Basic Engineering, vol. 94, no. 2, pp. 339-344, 1972.

[37] Bevilaqua P., Lee J., Development of a nozzle to improve the turning of supersonic Coanda jets, Tech. Rep. AFWAL-TR-80-3027, Air Force Wright Aeronautical Laboratories (Wright-Patterson Air Force Base), 1980.

[38] Favre-Marinet M., Binder G., HacT., Generation of oscillating jets, Journal of Fluids Engineering, vol. 103, pp. 609-614, 1981.

[39] Allen D., Axisymmetric Coanda-Assisted Vectoring. PhD thesis, Utah State University, 2008.

[40] Neuendorf R., Lourenco L., Wygnanski I., On large stream-wise structures in a wall jet owing over a circular cylinder, Physics of Fluids, vol. 16, pp. 21582169, 2004.

[41] Chiang T., Sheu T., Wang S., Side wall effects on the structure of laminar flow over a plane-symmetric sudden expansion, Computers \& Fluids, vol. 29, pp. 467-492, 2000.

[42] Nishino T., Hahn S., Shari K, Large-eddy simulations of a turbulent coanda jet on a circulation control airfoil, Physics of Fluids, vol. 22, pp. 125105-1-12510515, 2010.

[43] Dumitrache A., Frunzulica F., lonescu T., Nonlinearity, Bifurcation and Chaos - Theory and Applications, ch. Mathematical Modelling and Numerical Investigations on the Coanda Effect, pp. 101-132. INTECH, 2012.

[44] Payne P., Curved jet ows (vol i), Tech. Rep. USAAML 
Technical Report 65-20, US Army Aviation Material Laboratories, Fort Eustis, Virginia, USA, 1965.

[45] AGARD, Effects of streamline curvature on turbulent flow, Tech. Rep. AGARDograph N169, Advisory Group for Aerospace Research and Development, 1973.

[46] Kobayashi R., Fujisawa N., Curvature effects on twodimensional turbulent wall jets, Ingenieur-Archiv, vol. 53, pp. 409-417, 1983.

[47] Englar R., Experimental investigation of the high velocity coanda wall jet applied to bluff trailing edge circulation control airfoils, tech. rep., Naval Ship Research and Development Center (USA), 1975.

[48] Bevilaqua P., Lifting surface theory for thrust augmenting ejectors," tech. rep., Rockwell International Columbus OH (USA), 1982.

[49] Azim M., Sadrul-Islam M., Plane mixing layers from parallel of two streams, Experiments in Fluids, vol. 34, pp. 220-226, 2003.

[50] Matsuo S., Setoguchi T., Kudo T, Study on the characteristics of supersonic Coanda jet, Journal of Thermal Science, vol. 7, no. 3, pp. 165-175, 1998.

[51] Alvi F., Strykowski P., Krothapalli A., Forliti D., Vectoring thrust in multiaxes using confined shear layers, Journal of Fluids Engineering, vol. 122, no. 1, pp. 313, 2000.

[52] Cattafesta L., Sheplak M., Actuators for active flow control, Annual Review of Fluid Mechanics, vol. 43, p. 247272, 2011.

[53] Panzarella P., The use of a Coanda nozzle with parallel secondary injection for the thrust vectoring of a two-dimensional compressible fluid, Master's thesis, Air Force Institute of Technology (USA), 1965.

[54] Hanson R., Lavoie P., Naguib A., Morrison J., Transient growth instability cancellation by a plasma actuator array, Experiments in Fluids, vol. 49, pp. 1339 1348, 2010.

[55] Corke T., Post M., OrlovD., Sdbd plasma enhanced aerodynamics: concepts, optimization and applica- tions, Progress in Aerospace Sciences, vol. 43, p. 192217, 2007.

[56] Sosaa R., Artana G., Steady control of laminar separation over airfoils with plasma sheet actuators, Journal of Electrostatics, vol. 64, pp. 604-610, 2006.

[57] Orlov D. M., Modeling and simulation of single dielectric barrier discharge plasma actuators. PhD thesis, University of Notre Dame, 2006.

[58] Orlov D., Apker T., He, C. Othman H., et al., Modeling and experiment of leading edge separation control using sdbd plasma actuators, in AIAA 45th Aerospace Sciences Meeting, 2007.

[59] Lemire S., Vo H., Reduction of fan and compressor wake defect using plasma actuation for tonal noise reduction, Journal of Turbomachinery, vol. 133, pp. 133-143, 2011.

[60] Grundmann S., Frey M., Tropea C., Unmanned aerial vehicle (UAV) with plasma actuators for separation control, in 47th AIAA Aerospace Sciences Meeting, 2009.

[61] Thomas F., Kozlov A., Corke T., Plasma actuators for cylinder flow control and noise reduction, AIAA Journal, vol. 46, pp. 1921-1931, 2008.

[62] Whalley R., Turbulent Boundary-Layer Control with DBD Plasma Actuators Using Spanwise TravellingWave Technique. PhD thesis, University of Nottingham, 2011.

[63] Tornabene F., Ceruti A., Mixed static and dynamic optimization of four-parameter functionally graded completely doubly curved and degenerate shells and panels using GDQ method, Mathematical Problems in Engineering, vol. 1, p. 33, 2013.

[64] Liverani A., Ceruti A., Caligiana G., Tablet-based 3D sketching and curve reverse modelling, Inter-national Journal of Computer Aided Engineering and Technology, vol. 5, no. 2/3, pp. 188-215, 2013. 